\title{
Age Estimation And Gender Classification Based On Face Detection And Feature Extraction
}

\author{
Sasikumar Gurumurthy, C. Ammu , B. Sreedevi \\ School of computer science and Enginerring, VIT University, Vellore \\ g.sasikumar@vit.ac.in \\ School of computer science and Enginerring, VIT University, Vellore. \\ ammu.c2009@vit.ac.in \\ School of computer science and Enginerring, VIT University, Vellore. \\ Sreedevi861@gmail.com
}

\begin{abstract}
Nowadays the computer systems created a various types of automated applications in personal identification like biometrics, face recognition techniques. Face verification has turn into an area of dynamic research and the applications are important in law enforcement because it can be done without involving the subject. Still, the influence of age estimation on face verification become a challenge to decide the similarity of pair images from individual faces considering very limited of data base availability. We focus on the development of image processing and face detection on face verification system by improving the quality of image quality. The main objective of the system is to compare the image with the reference images stored as templates in the database and to determine the age and gender.
\end{abstract}

\section{Indexing terms/Keywords}

Eigen face, Fisherface, Histogram equalization, k-cross fold validation.

\section{Council for Innovative Research}

Peer Review Research Publishing System

Journal: International Journal of Management \& Information Technology

Vol.4, No.1

editor@cirworld.com

www.cirworld.com, member.cirworld.com 


\section{INTRODUCTION}

The importance of this paper is the design of image processing system and face recognition on face verification system to improve image quality with the purpose of identify the level similarity of face images based on the age stages and finding the gender of the persons.

The first step is Image processing where quality of face image is improved and enhanced using histogram equalization methods. Brightness Preserving Dynamic Fuzzy Histogram Equalization (BPDFHE) method [3] is used for increasing brightness and contrast enhancement and translation of color images into grayscale to get accurate result. Histogram equalization is mainly useful in images with foregrounds and backgrounds that are both dark or both bright. Especially, the method can direct to better outlooks of bone structure in x-ray images, and to better detail in photographs that are over or under-exposed. There are lots of techniques available for face detection. Here we used Image segmentation and image filling methods to detect the faces. The next step is Feature extraction which is preprocessing level for age estimation and gender verification. Feature extraction is specific form of dimensionality reduction in image processing and pattern recognition. we use an eigen face using PCA (Principal Component Analysis) method [5] to recognize the faces from the database. Eigen faces are group of Eigen vectors mainly used in computer vision. This approach was developed by Sirovich and Kirby. We used eigenface mainly for age estimation. For Gender classification we used Fisherface algorithm. It uses Principal of Linear Discriminant Analysis method. Using this we find out whether the given image belongs to the gender female or male.

The efficiency of the algorithms are checked using K-Cross fold validation and Leave one out classifiers. The classifiers when used over the few publicly available databases, gives out prominence results. The design of the system is showed in the figure 1. It has two main parts namely Enrolment and verification.

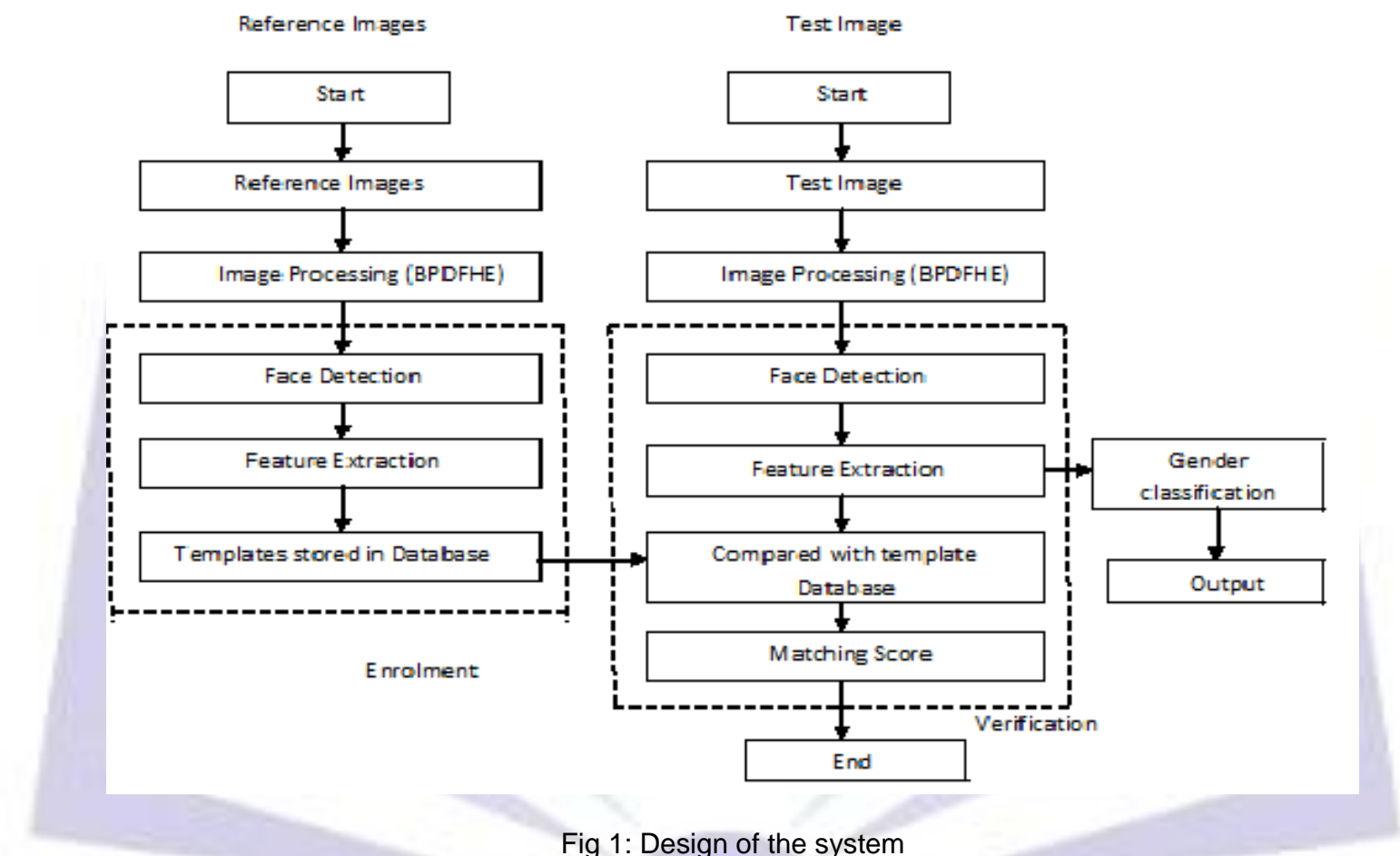

\section{METHODS AND TECHNIQUES}

\section{Histogram Equalization}

Histogram equalization is method of transforming contrast. This method is efficient because, it uses intensity values alone. We used Brightness Preserving Dynamic Fuzzy Histogram Equalization (BPDFHE) to improve its brightness and contrast enhancement abilities while decreasing its complexity of computation. This method uses fuzzy statistics of images for their processing and representation. In the fuzzy domain representation and processing of images allows the technique to handle the inaccuracy of gray level values in a better way, resulting in improved performance. Moreover, the inexactness in gray levels is handled well by fuzzy histogram, fuzzy statistics, when calculated with suitable fuzzy membership function, does not have random oscillations or missing intensity levels and is basically smooth. This helps in attaining its significant partitioning needed for brightness preserving equalization. The BPDFHE technique consists Fuzzy Histogram Computation, Partitioning of the Histogram, Dynamic Histogram Equalization of the Partitions, Normalization of the image brightness.

\section{Face Detection}

For Face Detection we used Techniques like Image segmentation and image filling to detect the faces. Initially the image is segmented by subtracting the image with the RGB values of the skin tone color. Then the image is turned to pure black 
where ever the color pixels are found after the subtraction of the skin tone. Then we get the image of faces alone. The noises in the image are filled with the white pixels by calculating the surrounding spaces intensity. If the surrounding pixels are of white then the center pixels are changed to white again. This should be done on a single loop alone.

At the last remaining low space dimensional areas of white pixels are removed used the image filling method. After the face regions are separated, the faces are surrounded with a bounded box with the face as a center and the image is cropped. This process is a pre-process for Eigenface recognition. These images are made into templates and sent as input for the Eigen Face recognition.

\section{FEATURE EXTRACTION}

\section{Eigenface Algorithm}

A set of eigen faces can be formed by performing a mathematical process called principal component analysis (PCA) on a huge set of images describing dissimilar human faces. The eigenface recognition system can be split into two main parts: creation of the eigenface basis and detection or recognition of a new face.

Creation of Eigen face:The first step is obtaining a set $\mathrm{S}$ with $\mathrm{M}$ face images. Every image is converted into a vector of size $\mathrm{N}$ and positioned into the set.

$$
S=\left\{\Gamma_{1}, \Gamma_{2}, \Gamma_{3}, \ldots \ldots \ldots \Gamma_{M}\right\}
$$

After obtaining a set, We need to obtain the mean image $\boldsymbol{\Psi}$

$$
\Psi=\frac{1}{M} \sum_{n=1}^{M} \Gamma n
$$

Then we find the difference $\boldsymbol{\Phi}$ between the given input image and the mean image $\boldsymbol{\Psi}$

$$
\Phi i=\Gamma_{i}-\Psi
$$

Next we look for a set of $M$ orthonormal vectors, $\mathbf{u}_{\mathbf{n}}$ and $k^{\text {th }}$ vector, $\mathbf{u}_{\mathbf{k}}$, such that

$$
\lambda_{k}=\frac{1}{M} \sum_{n=1}^{M}\left(u_{k}^{T} \Phi_{n}\right)^{2}
$$

is a maximum, subject to

$$
u_{i}^{T} u_{k}=\delta_{i k}=\left\{\begin{array}{l}
1 \quad \text { if } l=k \\
0 \text { otherwise }
\end{array}\right.
$$

Where $\boldsymbol{\lambda}_{\mathbf{k}}$ and $\mathbf{u}_{\mathbf{k}}$ are the eigenvalues and eigenvectors of the covariance matrix $\mathbf{C}$. We obtain the covariance matrix $\mathbf{C}$ using following formula

$$
\begin{gathered}
C=\frac{1}{M} \sum_{n=1}^{M} \Phi_{n} \Phi_{n}^{T} \\
=\mathrm{AA}^{\top}
\end{gathered}
$$$$
A=\left\{\Phi_{1}, \Phi_{2}, \Phi_{3}, \ldots \ldots, \Phi n\right\}
$$

Then $\mathrm{A}^{\top}$

$$
L_{m n}=\Phi_{n}^{T} \Phi_{n}
$$

we have found the eigenvectors, $\mathbf{u}_{\mathbf{l}}, \mathbf{v}_{\mathbf{I}}$

$$
u_{\mathbb{1}}=\sum_{k=1}^{M} v_{l k} \Phi_{k} \quad l=1, \ldots \ldots, M
$$

Recognition:Given input face image is transformed into its components of eigenface. Then we compare the input image with mean image and multiply their difference with each eigenvector of the $L$ matrix. Each value would represent a weight which would be stored on a vector $\Omega$. 


$$
\begin{gathered}
\omega_{k}=u_{k}^{T}(\Gamma-\Psi) \\
\Omega^{T}=\left[\omega_{1}, \omega_{2}, \ldots \ldots x \omega_{M}\right]
\end{gathered}
$$

Next step, determine which face class gives the best description for the given input image. This is through minimizing the Euclidean distance

$$
\varepsilon_{k}=\left\|\Omega-\Omega_{k}\right\|^{2}
$$

If $\varepsilon_{k}$ is bellow an established threshold $\theta_{\varepsilon}$, the input face is consider to belong to a class. Then the input face image is taken into account of a known face. If the difference is more than given threshold, but bellow a second threshold value, the image can be considered as a unknown face. If the input image is above those two threshold values, the image is determined NOT to be a face image.

\section{Comparison of age stages}
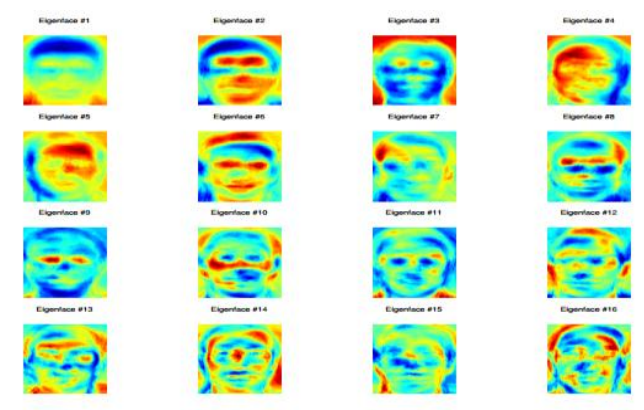

Fig2. Eigenfaces

In this step we use comparison algorithm to compare the test image with the reference image set stored in the database and the matching score is given as output. For the comparison, we need a database of images with their respective ages specified. Then each image is compared with the input images and the most common features available is selected and the age is determined.

\section{Fisherface Algorithm}

It designed using Fisher's Linear Discriminant Analysis which maximizes the ratio of between-classes to within-classes scatter. The plan is simple: same classes should cluster together tightly, while different classes are as far away as possible from each other. The algorithm is described as follows:

Construct the Image matrix $X$ with each column representing an image. Each image is a allocateed to a class in the corresponding class vector $\mathrm{C}$. Project $\mathrm{X}$ into the $(\mathrm{N}-\mathrm{c})$-dimensional subspace as $\mathrm{P}$ with the rotation matrix WPca recognized by a Principal Component Analysis, where $\mathrm{N}$ is the number of samples in $\mathrm{X}$ and $\mathrm{c}$ is unique number of classes.

Find the between-classes scatter of the projection $\mathrm{P}$ as,

$$
s_{W}=\sum_{i=1}^{e} N_{i} *\left(\text { mean } n_{i}-\text { mean }\right) *\left(\operatorname{mean}_{\mathrm{i}}-\text { mean }\right)^{\wedge} \mathrm{T}
$$

Where mean is the total mean of $P$, mean_ $i$ is the mean of class $i$ in $P$ and $N \_i$ is the number of samples for class $i$.

Calculate the within-classes scatter of $P$ as

$$
s_{W}=\sum_{i=1}^{e} \sum_{x_{k} \in X_{-} i}\left(x_{k}-\text { mean }_{i}\right) *\left(x_{k}-\text { mean_i }\right)^{\wedge} T
$$

Where $X \_i$ are the samples of class $i, x \_k$ is a sample of $X \_i$, mean_ $i$ is the mean of class $i$ in $P$.

Apply a standard Linear Discriminant Analysis and maximize the ratio of the determinant of between-class scatter and within-class scatter. The result is given by the set of simplified eigenvectors Wfld of $\mathrm{Sb}$ and Sw corresponding to their eigenvalue. The rank of $\mathrm{Sb}$ is atmost (c-1), so there are only (c-1) non-zero eigenvalues, cut off the rest.Finally obtain the Fisherfaces by

$$
W=W P c a * W f l d
$$




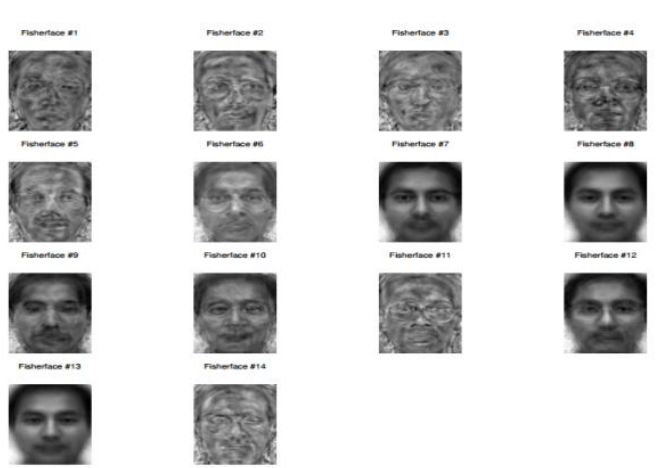

Fig3.Fisherface

\section{Gender classification}

After obtaining features via fisherface method, features of the training dataset will be compared with the features of input image to classify whether the given input image is female or male gender.

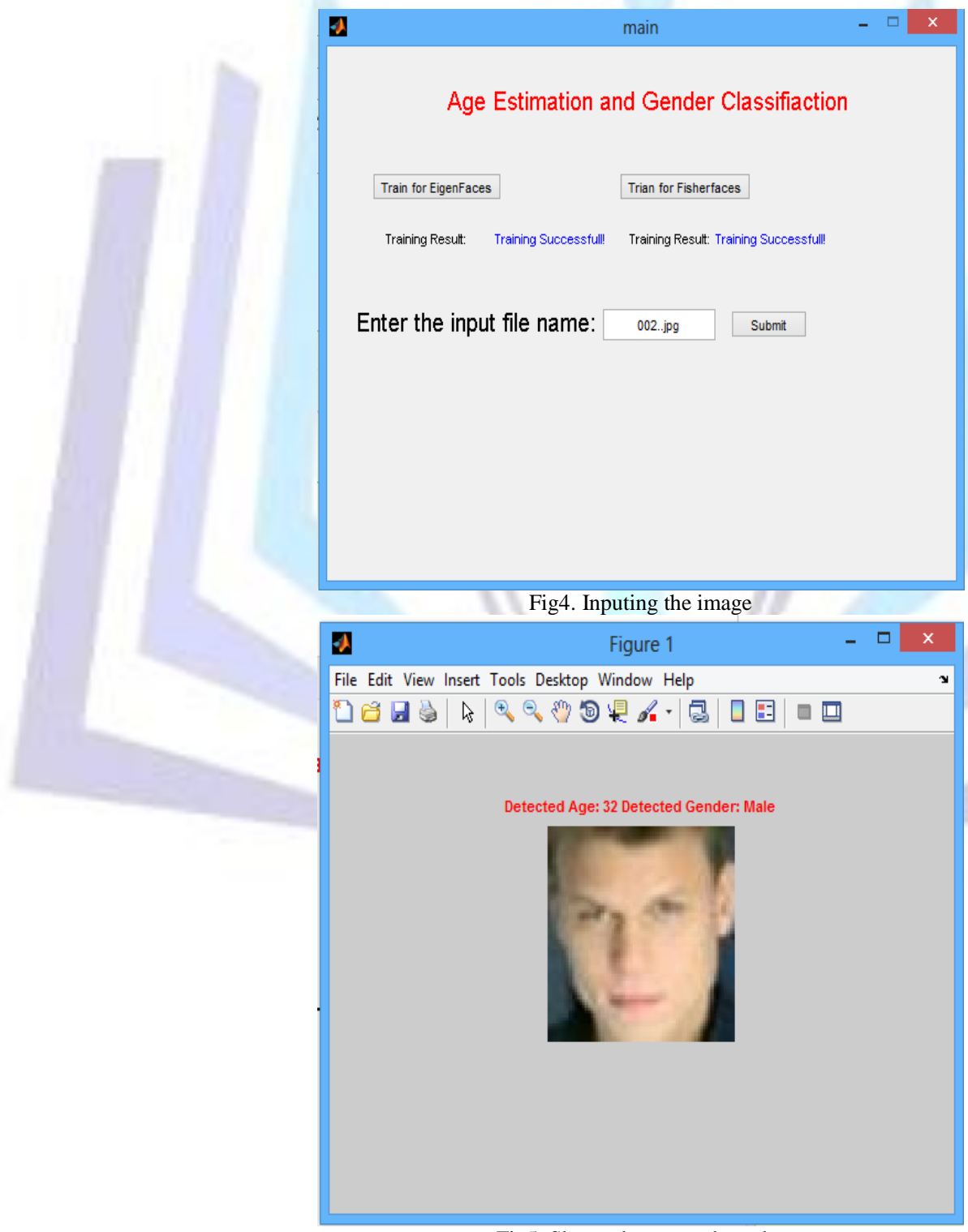

Fig5. Shows the age and gender 


\section{RESULTS}

The experimental results demonstrate that the accuracy of the prediction increases when the dataset provided for the training increases.

The algorithms we have discussed so far have been implemented using the MATLAB and has been tested with many different possible datasets which are available publicly in the internet. These datasets for images vary with different constraints like illumination, face expression and many other things like pose etc., The dataset is trained with different types of datasets because it can be used in any generic application. The implementation is then tested with many possible constraints to check for accuracy and errors. And the results have been really prominent.

\section{Eigen Faces:}

We perform the analysis based on the Leave one class out classifier.

The recognition rate was found to be $83.5 \%$ and we are using 165 models. And each model needs a computation need of 3 seconds and which leads a total computation of 8 minutes.

Error rate and reduced space:

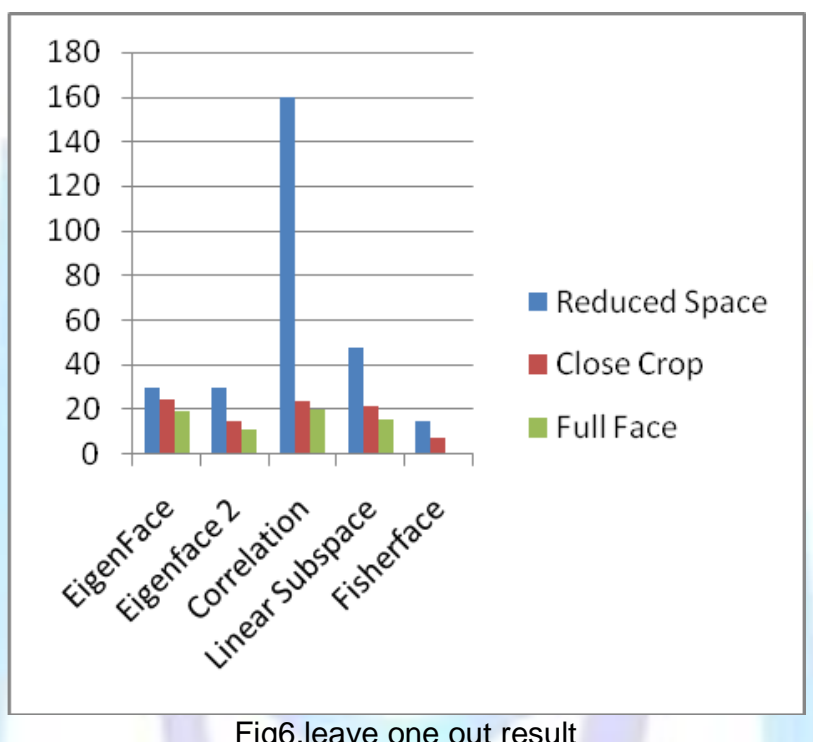

Then we perform the analysis with the K-fold cross validation. The K-cross fold validation is performed with 5 folds on dissimilar no. of components. The recognition rate is as follows.

10 components: $73.33 \%$

25 components: $80.00 \%$

40 components: $80.67 \%$

55 components: $81.33 \%$

70 components: $82.67 \%$

85 components: $81.33 \%$

100 components: $82.00 \%$

Error rate on yales database:

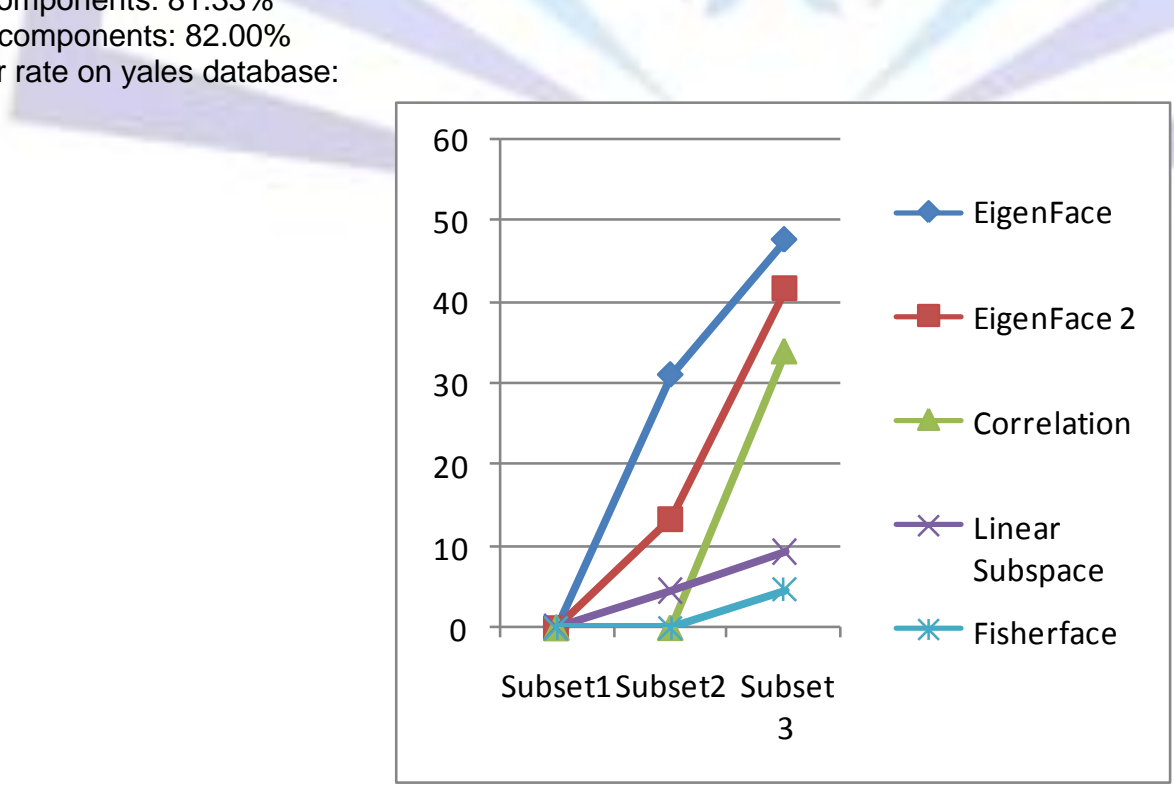

Fig7. K-cross fold result 


\section{CONCLUSIONS}

On this dataset the Fisherface method is performing much better, than the Eigenfaces method .It yields a much better recognition performance, but the ability to reconstruct faces from the Eigenspace is gone. So we can conclude that the both methods are complementing each other. It would be a good suggestion to keep the PCA object around (already computed in the Fisherfaces method) so as to use its reconstruction capabilities.

\section{Age Synthesis:}

Age synthesis is a developing concept, which needs more developers and researchers on it. The basic concept is to predict the image model of certain person on basis of age which does not even exist. Consider we have a 20 years photo of a subject and from this image we can predict what he will be like in 40 years. To make it possible we use same algorithms which are described in this paper but the dataset has to be more volatile. And it should contain large no. of images which are rather again divided on basis of countries so that the age recognition can be more accurate. To perform analysis on databases these large takes more time and processing units.

\section{REFERENCES}

[1] Nana Rachmana Syambus and Untung Hari Purwanto: "Image Processing and Face Detection Analysis on Face Verification Based on the Age Stages" $7^{\text {th }}$ International Conference on Telecommunication systems, Services and Applications, 2012.

[2] Debdoot Sheet, Hrushikesh Garud, Amit Suveer, Manjunatha Mahadevappa "Brightness Preserving Dynamic Fuzzy Histogram Equalization", IEEE Transactions on Consumer Electronics, Vol. 56, No. 4, November 2010

[3] Abdul Samad Khan and Lawang Khan Aliza: "Introduction to Face Detection Using Eigenfaces", IEEE-ICET,2006.

[4] BilginEsme \& BulentSankur BogaziçiUn : "Effects of Aging over Facial Feature Analysis and FaceRecognition", Electronics Eng. Dept. March 2010.

[5] TangquanQi , Huiwen Deng and Weiping Hu: "Face Recognition using Eigenfaces-Fisher Linear Discriminant and Dynamic Fuzzy Neural Network", $3^{\text {rd }}$ International conference on Computer Science and Information Technology (ICCSIT), 2010.

[6] Hossein Sahoolizadeh and Youness Aliyari Ghassabeh: "Face Recognition using Eigen-faces, Fisherfaces and Neural Networks", 7th IEEE International Conference onCybernetic Intelligent Systems, 2008.

[7] N. Ramanathan and R. Chellappa : Face Verification Across Age Progression, IEEE Transactions on Image Processing, Vol. 15, No. 11,2006.

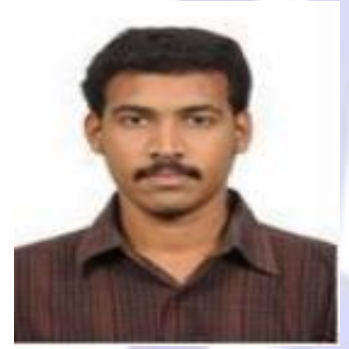

Sasikumar Gurumurthy is an assistant professor (Sr.) in SCSE, VIT University, Vellore, Tamil Nadu, India. He received B.E Degree in Computer Science and Engineering from Kamaraj University, Madurai in 2003 and M.E Degree in Computer Science \& Engineering from Anna University, Chennai in 2005. He has published more than 70 technical papers in international journals proceedings of international conferences. He is having more than 8 years of teaching Experience. He is a member of international professional associations like CSI, IAENG, AIRCC, MHRO and is a reviewer of around 2 international journals. He is currently doing his Phd in VIT University. His current fields of research interest include image processing, signal processing and bio-medical engineering. 